\title{
Can Breast Cancer Survivors Benefit from Speed of Processing Training? A Perspective Article on Treatment and Research
}

\author{
Kristen Triebel ${ }^{1}$ \\ Joseph Anderson (iD ${ }^{2}$ \\ Sai Rashmi Nakkina ${ }^{3}$ \\ David E Vance (D) ${ }^{4}$ \\ 'Department of Neurology, Division of \\ Neuropsychology, Birmingham, AL, USA; \\ ${ }^{2}$ Department of Neurology, University of \\ Alabama at Birmingham, Birmingham, AL, \\ USA; ${ }^{3}$ College of Arts and Science, \\ University of Alabama at Birmingham, \\ Birmingham, AL, USA; ${ }^{4}$ School of \\ Nursing, University of Alabama at \\ Birmingham, Birmingham, AL, USA
}

Correspondence: David E Vance School of Nursing, University of Alabama at Birmingham, I70I University

Boulevard, Birmingham, AL, 35294-1210, USA

Tel +205-934-7589

Fax +205-996-7I83

Email devance@uab.edu

\begin{abstract}
For breast cancer survivors (BCS), issues related to quality of life after treatment have become increasingly important. Up to $75 \%$ of individuals receiving chemotherapy experience cognitive impairment and for many BCS, these impairments persist for a long time after treatment. In addition to these cognitive impairments, research suggests a higher rate of depression, anxiety, and distress in cancer survivors. The impact of these cognitive and emotional deficits impairs daily functioning, occupational performance, increases relationship stress and caregiver burden, contributes to poor emotional processing, and reduces quality of life. Fortunately, there are cognitive interventions that can improve targeted cognitive abilities along with general cognitive processes. Speed of processing (SOP) training, a type of cognitive remediation therapy, has been shown to improve cognitive performance on measures of visual attention, SOP, and timed-task performances. Furthermore, SOP has been shown to enhance neuromodulatory systems that improve mood, especially depression and other indicators of quality of life. Studies support the need for deeper investigation into the short-term and long-term benefits of SOP training as a behavioral adjunct for BCS.
\end{abstract}

Keywords: cognitive training, cognitive remediation, cognitive aging, breast cancer survivors

In the United States, there were more than 3.5 million women living with a history of breast cancer. ${ }^{1}$ This figure includes women currently being treated and women who have finished treatment. Advances in breast cancer treatment have dramatically increased survival as evidenced by 5-year survival rates in the United States at approximately $90 \%$ and 1.4 million breast cancer survivors (BCS) more than 20 years post-diagnosis. ${ }^{2}$ With BCS living longer, increased attention has been paid to the residual effects of treatment. ${ }^{3,4}$ One of the more common side effects is cognitive impairment, which affects up to $90 \%$ of persons with breast cancer ${ }^{5,6}$ and can persist for a long time after treatment is completed. ${ }^{7-9}$ Speed of processing (SOP), executive functioning, attention, and memory are particularly affected. ${ }^{7,10,11}$ These cognitive difficulties can negatively impact everyday functioning which can be very distressing to BCS. ${ }^{3,12-14}$

Despite the high risk for cancer-related cognitive impairment among BCS, there are few treatments available to remediate these deficits. A study by Meneses et $\mathrm{al}^{15}$ found promising effects of a computerized SOP intervention for middle- to olderaged women with breast cancer. Findings from studies of healthy older adults who 
received SOP training suggest that the training may have secondary benefits to mood, internal locus of control, everyday functioning, and quality of life. ${ }^{16-18}$

The purpose of this article is to provide a rationale for why SOP training, a type of cognitive training, is particularly suited as a possible therapeutic approach for treating cognitive problems in BCS. Figure 1 provides a graphic representation of this argument. Specifically, on the left are issues experienced by BCS and on the right are known therapeutic benefits of SOPs training with the instruments in which these benefits are measured listed in the middle of the figure. In providing this rationale, first a background of cognitive training is provided. Second, from all the cognitive trainings, a description of SOP training is provided, including information on its benefits in improving cognitive, everyday functioning, and noncognitive outcomes, including its neurological underpinning. Third, the cognitive and non-cognitive deficits observed in BCS are described and matched as to how SOP training can address each deficit. Finally, implications for research of how SOP training can be suited in this clinical population are provided.

\section{Cognitive Training}

Cognitive training refers to a therapeutic approach in which an assortment of stimulating activities, mental exercises, and thinking strategies are employed with the expressed purpose to improve cognitive abilities. The principle supporting cognitive training centers on the concept of neuroplasticity. By and large, neuroplasticity refers to the brain's ability to form new and stronger connections between neurons and brain regions in response to being exposed and adapting to challenging and novel environmental stimuli (eg, playing a game, joining a club). ${ }^{19-22}$ Thus, the biochemistry, metabolism, and morphology of the brain are changed in forming these connections, allowing specific types of information to be processed more efficiently. ${ }^{19,21}$ In examining the efficacy and utility, the types and methods of administering cognitive training varies greatly from study to study. Some types are composed of small classes with pencil and paper tasks that require participants to mentally process information in a specified, prescribed manner. Other types include completing workbooks, while others employ watching prerecorded assignments. ${ }^{23}$ Dosage also varies greatly with some studies delivering the training in a single session to

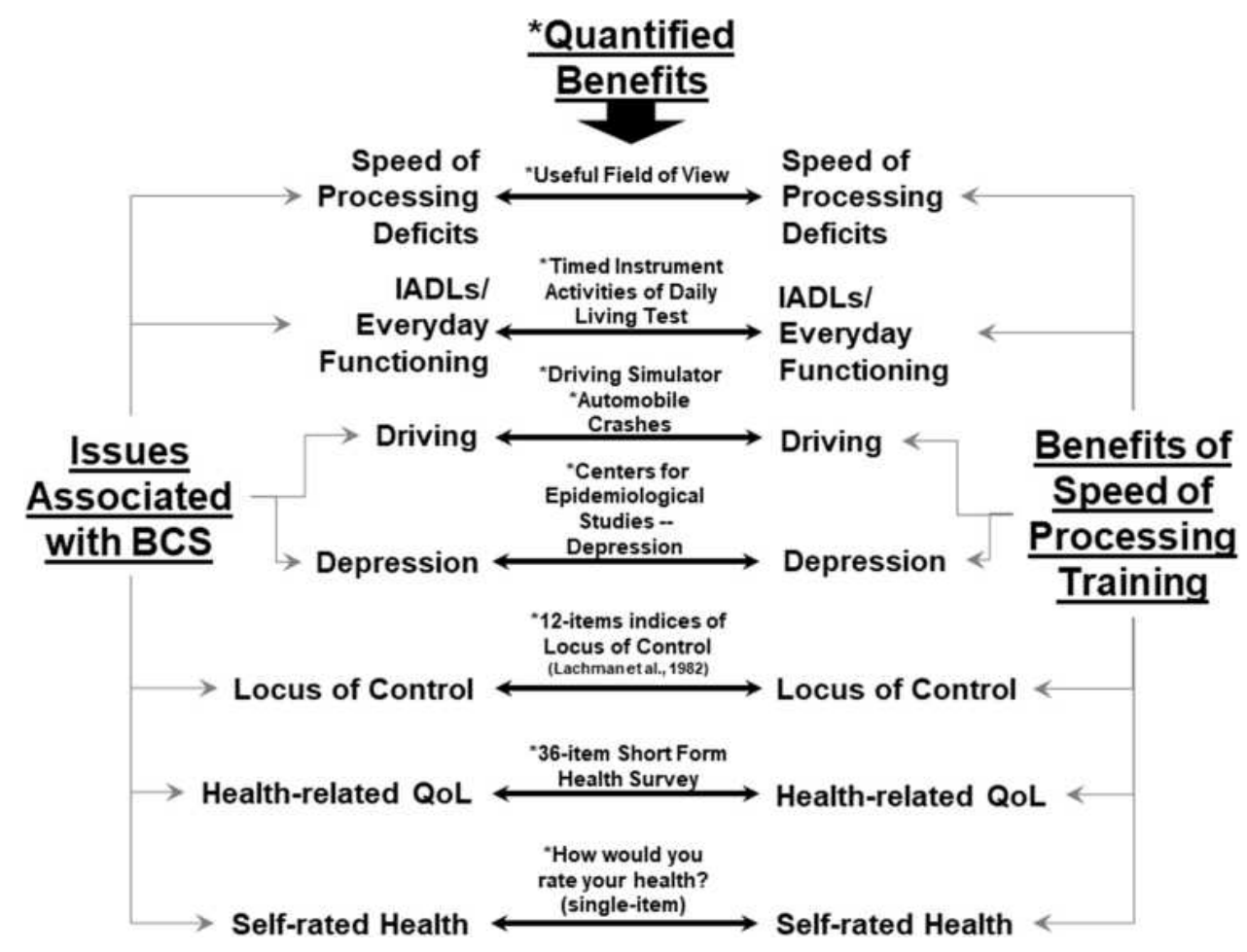

Figure I Domains in which SOP training may benefit BCS. Asterisks indicate instruments/measures that have been used in the literature to quantify these benefits. This figure was developed by the David Vance and is the property of this author. 
others requiring up to 30 hours of training exposure; however, a common dosage observed in the literature is 10 hours of training. Location also is a consideration as some studies administer the cognitive training in a controlled laboratory setting to monitor treatment fidelity and maintain internal validity while others administer it in the community or the participant's home under realistic conditions, thus strengthening the ecological validity of the study. Yet, with the sophistication of gaming software and the widespread use of computers and smart devices, computerized cognitive training approaches have gained prominence especially in older adults and clinical populations concerned with improving or protecting their cognitive abilities. ${ }^{20,24}$

Cognitive training approaches can target global cognitive function as well as specific cognitive domains such as attention, working memory, verbal memory, nonverbal/ spatial memory, reasoning/executive functioning, and SOP. In fact, many cognitive training approaches focus on delivering stimuli targeting improvement or remediation of such a specific cognitive domain versus the more daunting task of trying to improve global cognition. In a systematic meta-analytic study of 52 computerized cognitive training studies targeting older adults $(60+$ years $)$, Lampit, Hallock, and Valenzuela examined the efficacy of different types of computerized cognitive training to improve a variety of cognitive domains that were targeted for treatment. ${ }^{25}$ Pooling the effect sizes across studies, cognitive improvements from computerized cognitive training were robustly observed for SOP $(g=0.31)$, visuospatial skills $(g=0.30)$, nonverbal memory $(g=$ $0.24)$, working memory $(g=0.22)$, and verbal memory $(g=0.08)$. Although some of the individual studies did find significant improvements in attention and executive functioning following cognitive training, collectively the pooled effected sizes were not significant.

Included in this meta-analysis is the ACTIVE (Advanced Cognitive Training for Independent and Vital Elderly) Study, the largest longitudinal clinical trial of cognitive training. In the ACTIVE Study, 2,802 normal community-dwelling older adults from six sites across the United States were randomly assigned to one of four groups: 1) a memory training group; 2) a reasoning training group; 3) a SOP training group; and 4) a no-contact control group. Those in the training groups received 10 hours of laboratory training spread over a 4-6 week period. One of the primary findings from the ACTIVE Study is that with only 10 hours of cognitive training, participants improved in the cognitive domain in which they were trained; these cognitive training gains were more robust for reasoning and SOP training. In fact, based on the ACTIVE data, the US National Institute of Nursing Research and the US National Institute on Aging declared that SOP facilitates the ability for "older people to maintain their cognitive abilities as they age," even a decade after training. ${ }^{26}$ Using these longitudinal data, Edwards et al observed that compared to the no-contact control group, participants in the SOP training group experienced a $29 \%$ reduced risk of dementia over a 10 year period. ${ }^{27}$ Thus, based on the Lampit et al's metaanalysis as well as these findings from the ACTIVE Study, SOP training may be a particularly therapeutic approach to improve and protect cognition and everyday functioning. ${ }^{25}$

\section{SOP Training}

SOP training described in the literature mostly refers to the training protocol used in the ACTIVE Study and then later adapted into a software package by PositScience, Inc. (brainhq.com). Although there are several types of SOP training exercises within this software package, Divided Attention is generally considered the most representative of them. ${ }^{28-30}$

In Divided Attention, a repeated series of small trials are presented to participants. In a given trial, participants are directed to stare at the computer screen during which a central target (type of vehicle - car or truck) and a peripheral target (Route 66 sign) are presented simultaneously and maintained on the screen from around 17 to $500 \mathrm{~ms}$ (Figure 2A). Immediately after this brief exposure, participants are given a choice between two vehicles and asked to select the vehicle they just saw (Figure 2B); in this example, a car. If they are incorrect, a clunking sound is presented which indicates they responded incorrectly and then they progress to the next trial. If they chose correctly, the next screen prompts them to select the location of the peripheral target (Figure 2C); in this example, they are to click in the middle-upper left lightly shaded area. If they chose incorrectly, again a clunking sound is presented indicating the response is wrong and then they progress to the next trial. If they chose correctly, then a congratulatory sound is presented along with flashing stars and points awarded. After this, the participants are presented another trial and this repeats. Within an hour of training, participants repeat this process hundreds of times in which they are rewarded for correct responses. An 

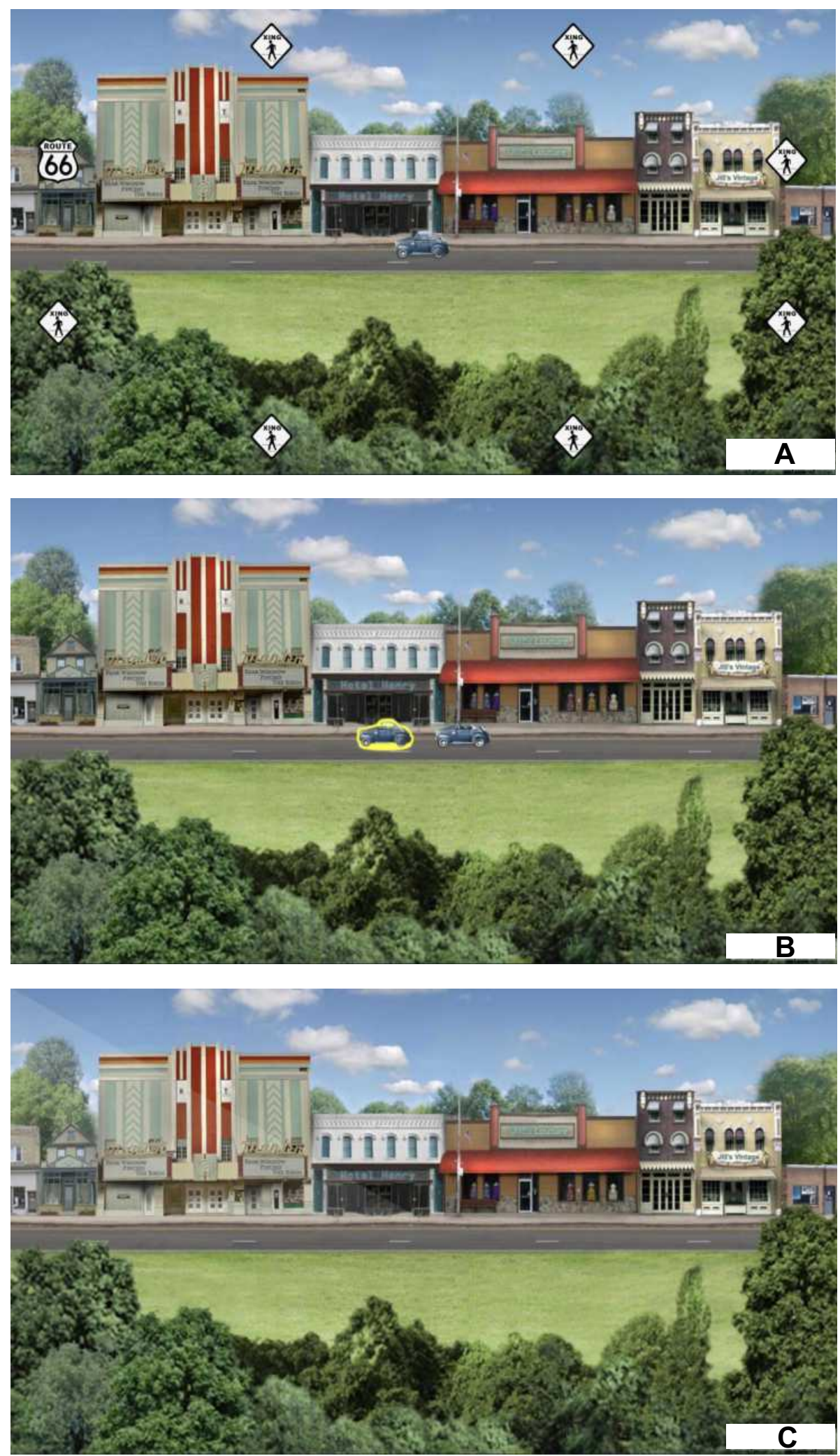

Figure 2 (A) Divided Attention with a Central and Peripheral Object. This figure was provided by Posit Science, Inc. and they have provided permission to use this figure. (B) Divided Attention with a choice to select the Central Object. This figure was provided by Posit Science, Inc. and they have provided permission to use this figure. (C) Divided Attention with a choice to select the location of the Peripheral Target. Once the participants chose the correct vehicle that was just presented from the choice provided in the left panel, then using the computer mouse, participants indicate where the outside object was present by clicking within that area. If they get it right, they get rewards with a little "ding" sound; if they get it wrong, they are punished with a little "clunk" sound. This figure was provided by Posit Science, Inc. and they have provided permission to use this figure. 
important component of this process is that if participants respond incorrectly, the next trial is made easier by slowing down the presentation speed of the target stimuli, and if participants respond correctly, the next trial is made harder by increasing the presentation speed. Thus, an algorithm is in place that allows participants to respond correctly approximately $75 \%$ of the time in order to encourage and motivate and avoid non-adherence in the participants, but yet always be challenging to ensure that neuroplastic changes are occurring in the brain.

\section{Improvement of SOP and Cognition}

In addition to the ACTIVE Study, other studies show that SOP training improves this cognitive ability. ${ }^{31}$ In a sample of 67 older adults 59-95 years old, Edwards et al randomized these participants into either the SOP training group $(n=36)$ or a wait list control group $(n=37) .{ }^{32}$ Over a 10 12 -week period, those in the SOP training group attended 2-3 sessions per week where they self-engaged the BrainHQ program (BrainHQ is a registered trademark of Posit Science, Inc.) with the goal of completing 20 1-hour sessions. During these sessions, participants engaged in a variety of visual/cognitive exercises designed to promote SOP. As in the other SOP training protocols, these exercises are adaptive as they display speeds and difficulty conforming to the participants' individual performance. As expected, compared to the wait list control group, the SOP training group experienced significant improvements on a visual SOP measured called Useful Field of View $\left(\mathrm{UFOV}^{\circledR}\right)$.

\section{Neurological Underpinnings of SOP Training}

From a neurological perspective, the characteristics of SOP training may uniquely alter the function and structure of the brain. Researchers have posited that the visual/ cognitive exercises of SOP training require one to process stimuli through repetition and sensory motor elaboration. Thus, repetitive procedural tasks are known to exert a wider array of regional brain activation than reasoning and memory tasks. ${ }^{33}$ Corroborating this, electrophysiological studies demonstrate that compared to controls, adults who receive SOP training exhibit increased P3b and N2pc amplitudes, an indicator of attentional allocation and capacity enhancement. ${ }^{34}$ This is important because deficits in inhibition and attentional control accompany prefrontal dysregulation, especially in BCS, which is an inefficient way to process information accurately and quickly. $8,35,36$ Thus, by reducing dependence on frontally oriented activity, SOP training promotes processing such information to posterior brain regions, thus improving SOP ability which translates to everyday functioning. ${ }^{13,16}$

In a pre-post MRI study of 33 healthy older adults (65+ years), Ross et al randomized participants into one of three groups: 1) 10 hours of SOP training using adaptive $\mathrm{UFOV}^{\circledR}$ training (similar to the Double Decision task developed by Posit Science, Inc.); 2) 10 hours of cognitively stimulating activities; or 3 ) a no-contact control. ${ }^{37}$ As expected, those in the $\mathrm{UFOV}^{\circledR}$ training group improved significantly in visual SOP. Compared to baseline, at posttest those in the $\mathrm{UFOV}^{\circledR}$ training group exhibited reduced brain activity in several regions of interest: anterior cingulate cortex, anterior insula, dorsolateral prefrontal cortex, inferior parietal lobe, supplementary motor area, and the thalamus. Similar results were not observed in the other two control groups. Furthermore, a large and significant increase in network connectivity was observed from baseline to post-test in the UFOV ${ }^{\circledR}$ training group. In fact, in examining 28 possible network connections, four network connections significantly showed increased strength: 1) anterior insula - supplementary motor area; 2) anterior insula - visual cortex; 3) anterior insula - supplementary motor area; and 4) dorsolateral prefrontal cortex - supplementary motor area. Based on these findings, it is suggested that increased connectivity improves processing efficiency which places less demand on brain regions to perform tasks. In fact, many of the resting-state functional connectivity with many of the regions of interest were strengthened following SOP training, thus enhancing visual attention efficiency and executive function.

\section{Improvement in Everyday Functioning}

The evidence on SOP training demonstrates that this type of cognitive training is not only effective for improving cognition, it yields gains in everyday functioning as well. Studies show that SOP training improves everyday functioning, particularly how quickly and accurately instrumental activities of daily living are performed. ${ }^{38}$ In fact, these outcomes have been replicated whether the SOP training occurs in the laboratory or at home. ${ }^{23,31,39}$

In numerous studies, a particular benefit of SOP training has been observed with automobile driving. Using the ACTIVE data, compared with a no-contact control group and a memory training group (ie, an active control), greater 
time engaged in SOP training was associated with maintenance of driving frequency over a 5 -year period. ${ }^{40}$

Similarly, over a 10-year period, after SOP training participants at-risk for driving mobility declines were $49 \%$ less likely to stop driving. ${ }^{30}$ Furthermore, when examining state-recorded motor vehicle collisions over nearly a 6-year period after training, those in the SOP training group experienced nearly a 50\% lower rate (per person-mile) of at-fault motor vehicle crashes. ${ }^{17}$ The benefits of improving visual attention and visual SOP through this particular training paradigm obviously has very real world applications. It is anticipated that BCS undergoing SOP training will experience improvements in driving ability (reduced accidents, fewer traffic violations, etc.) compared to those BCS who do not receive SOP training.

\section{Improvement in Non-Cognitive Outcomes}

From the ACTIVE Study, other health-related outcomes have been observed with this training including reduced severity of depressive symptomatology, ${ }^{41}$ better internal locus of control, ${ }^{42,43}$ self-rated health, ${ }^{44}$ and better healthrelated quality of life. ${ }^{45,46}$ Furthermore, compared to the reasoning training and memory training groups, those in the SOP training group retained these health-related outcomes up to five years after training.

In examining depression in particular, Wolinsky et al found that compared to the no-contact control group, those who received the SOP training experienced less clinically significant increases in depressive symptoms, as defined by $\geq 1.0$ standard deviation threshold on the Center for Epidemiological Studies Depression scale (CES-D)-12, by $30 \% 1$ year $(A O R=0.699, p=0.039)$ and 5 years $(A O R=0.651, p=0.059)$ after training. ${ }^{42}$ Employing the same statistical approach, these beneficial outcomes were not observed for the memory training or the reasoning training groups. In a separate but similar study examining 10 hours of laboratory SOP training versus 10 hours of attention control (ie, playing crossword puzzles), those in the SOP training group also had improved odds of experiencing reduced levels of depressive symptoms 1-year after training. 39

Neurologically, within the procedural activities of SOP training that require constantly focusing on stimuli presented and receiving feedback as to whether one's responses are correct, this may activate the attentional reward systems which enhances neuromodulatory system function; thus, this creates neuroplastic changes in brain morphology, enhancing brain functions accompanying mood. ${ }^{47-49}$ As mentioned, given that SOP training is programmed to present stimuli faster or slower to prevent one from becoming discouraged from responding wrong too much, an algorithm was placed into some of these programs to allow the program to speed up or slow down so that the participant gets about $75 \%$ of their answers correct; this then activates the reward system in the brain. In this manner, participants are rewarded for responding correctly but still cognitively challenged which encourages neuroplasticity. ${ }^{19,22}$

Since studies on SOP training suggest that it may protect one from developing depressive symptomatology, SOP training may be used in combination with pharmacological and cognitive-behavioral therapies as an adjuvant therapy to treat and possibly prevent depression. Likewise, as seen in the ACTIVE Study, by protecting one from developing depressive symptomatology, this may change one's perception, thus augmenting one's sense of locus of control, self-rated health, and health-related quality of life - an apparent benefit but unexpected benefit of SOP training.

\section{Utility SOP Training in Other Clinical Populations}

In the ACTIVE Study and others, SOP training has been shown to be a non-pharmacological and reasonably priced intervention that can effectively improve this cognitive ability in normal, community-dwelling older adults as well as in other clinical populations such as patients with HIV (eg, mild cognitive impairment). ${ }^{31,50,51}$ For example, in a sample of 46 middle-aged and older adults ( $40+$ years) with HIV, Vance et al randomized participants to either a 10 hours of SOP training group or a no-contact control group. ${ }^{51}$ Compared to the no-contact control group, those in the SOP training group improved on a measure of visual SOP and a laboratory measure of everyday functioning (ie, Timed Instrumental Activities of Daily Living test). Based on these favorable results, an ongoing 2-year longitudinal study is now investigating the effects of 10 versus 20 hours of SOP on middle-aged and older adults (40+ years) with HIV-Associated Neurocognitive Disorder. ${ }^{52}$ It is hypothesized that more SOP training will not only improve visual SOP and attention, but also driving outcomes (ie, reduced at-risk crashes, improved driving simulation outcomes), depression, locus of control, self-rated health, and healthrelated quality of life. 


\section{Cognitive Relevance to BCS}

Most women with breast cancer (without brain metastasis) experience cognitive impairment at some point in their disease trajectory. Up to $30 \%$ experience cognitive impairment prior to any treatment, $75 \%$ of patients with cancer experience cognitive impairment during treatment, and up to $35 \%$ of $\mathrm{BCS}$ experiencing cognitive problems years following systemic treatment. ${ }^{9}$ The exact mechanisms of cognitive impairment related to cancer and its treatment are unknown but hypothesized mechanisms include brain barrier alterations, cytokines and hormonal deregulation, inflammation, direct neurotoxicity of chemotherapy agents, ${ }^{9,53,54}$ and accelerated aging due to increased oxidative stress and decreased mitochondrial function. ${ }^{55}$

The cognitive abilities that are most vulnerable to impairment are the same abilities commonly affected by normal aging. Thus, slowed SOP is among the more commonly reported impairments found in BCS and is more pronounced in older BCS and especially in those treated with cytotoxic chemotherapy regimens. ${ }^{7,10,56,57}$ The finding that SOP is particularly affected in BCS provides support for the accelerated aging hypothesis. Hurria et al investigated cognitive impairment using neuropsychological tests in a group of older BCS (median age of 71 years) before chemotherapy and 6 months after chemotherapy. ${ }^{58}$ The prevalence of cognitive impairment at 6 months was $39 \%$, a statistic similar to studies of younger BCS. In contrast, other investigators have found interactions between age and chemotherapy on cognitive functioning. Adams-Price et al investigated treated and untreated BCS aged 39-71 years old and age-matched cognitively normal controls. ${ }^{56}$ They found that older BCS treated with chemotherapy performed significantly worse than the control group and the younger BCS treated with chemotherapy, but only on the SOP component of the UFOV ${ }^{\circledR}$ test. Ahles et al administered neuropsychological testing to a group of younger and older BCS treated with or without chemotherapy. ${ }^{59}$ These researchers found that older BCS who were treated with chemotherapy who had less cognitive reserve (as measured by a word reading test - Wide Range Achievement Test- 3) performed worse on SOP tests compared to older BCS who were not treated with chemotherapy. Thus, SOP seems to be a sensitive marker of cognitive impairment, particularly in vulnerable BCS (older age, chemotherapy treated, lower cognitive reserve) and is a cognitive impairment that could likely be improved with SOP training.

\section{Functional Relevance in BCS}

BCS often report having difficulty performing everyday tasks due to their cognitive impairments, although the scientific literature on the effects of cancer and cancer treatment on everyday functional tasks is sparse. One commonly reported difficulty is the ability to return to work. In recent meta-analysis of 40 studies, the overall rate of return to work among all cancer survivors was $57 \% .{ }^{60}$ A study by Von Ah et al found that self-reported decreased cognitive function was significantly correlated with worse work ability, performance, and productivity based on questionnaires given to the patients. ${ }^{61}$ Sun et al conducted a review of the literature and reported that the majority of the studies $(N=25)$ reported that BCS experienced reduced work engagement and work ability. ${ }^{62}$

Driving is another area of potential concern for BCS given the demands of SOP and attention resources necessary for safe driving. Adam-Price et al (2009) conducted a study examining $38 \mathrm{BCS}$ ( 3 to 45 months post chemotherapy) and compared them to 55 age-matched control participants in the performance on the $\mathrm{UFOV}^{\circledR}{ }^{56}$ $\mathrm{UFOV}^{\circledR}$ was used as a proxy for driving ability as studies have indicated $\mathrm{UFOV}^{\circledR}$ and driving abilities in older adults are highly correlated. ${ }^{63}$ These researchers found that older BCS performed more poorly than healthy agematched controls on the $\mathrm{UFOV}^{\circledR}$ SOP. Older BCS also performed more poorly than younger BCS. These researchers concluded that older BCS likely experience brain changes thought to be caused by chemotherapy because they are already at risk for behavioral slowing. Furthermore, this study raised concerns about driving performance, particularly among older BCS treated with chemotherapy. Future studies need to directly assess driving performance in BCS using driving simulators or onroad assessment.

\section{Aging and Cognition in BCS}

Although BCS of any age are vulnerable to cognitive impairment and SOP declines, there is a strong link between aging and cancer-related cognitive impairment. ${ }^{54,64,65}$ Aging shares many common biologic pathways with putative mechanisms of cancer-related cognitive decline. ${ }^{66}$ Chemotherapy also produces changes in biomarkers and brain structures that mimic aging. ${ }^{67-71}$ Studies have found that risk factors for cancer-related cognitive impairment include aging and age-related factors such as frailty, ${ }^{72,73}$ genetic risk factors (eg, apolipoprotein 
E-4 genotype), ${ }^{73}$ and high medical comorbidity burden (diabetes and cardiovascular disease). ${ }^{74}$

The Thinking and Living with Cancer study was a national, multisite prospective study that examined cognitive functioning in women with breast cancer who were aged 60 years or greater prior to receiving systemic treatment and then followed them over two years. ${ }^{74}$ This study identified several predictors of pre-treatment cognitive impairment in a sample of 164 women with nonmetastatic breast cancer. Cancer burden (or stage of disease) was associated with pre-treatment cognitive performance in that those with stage II or III breast cancer had lower executive functioning test scores compared to those with stage 0 to I breast cancer after adjusting for age, race, education, site of tumor, and surgery. ${ }^{74}$ In addition, women BCS who were older, nonwhite, less educated, and had greater medical comorbidity had greater odds of cognitive impairment before treatment. Medical comorbidities associated with pre-treatment impairment were diabetes and cardiovascular disease. Interestingly, depression, anxiety, fatigue, and surgery were not associated with impairment before treatment.

Using an expanded sample from the Thinking and Living with Cancer study ${ }^{75}$ that included 344 women with newly diagnosed nonmetastatic BCS and 347 matched controls without cancer, ${ }^{73}$ participants were assessed prior to treatment and at 12 and 24 months postsystemic treatment. BCS were divided into three treatment groups: 1) chemotherapy without hormonal therapy, 2) chemotherapy with hormonal therapy, and 3) hormonal therapy alone. Treatment was related to longitudinal cognitive performance; BCS who received chemotherapy had worse attention, SOP, and executive functioning test performance and those initiating hormonal therapy had lower learning and memory test performance compared to the other groups. These researchers also examined whether apolipoprotein E (ApoE) genotype (positive for e4 allele vs no e4 allele) affected the treatment group differences in cognitive scores over time. This variable was examined because individuals who have the ApoE e4 subtype are at greater risk for developing Alzheimer's disease. Interestingly, only BCS who had the ApoE e4 genotype (one or two alleles) receiving hormone therapy had shortterm decreases in learning and memory test scores. The attention, SOP, and executive function test scores were significantly worse for survivors positive for the ApoE e4 genotype who were exposed to chemotherapy. Finally, frailty was associated with self-reported cognitive decline but not with declines on cognitive testing.

In summary, BCS of all ages are vulnerable to SOP declines ${ }^{54,64,65}$ but older BCS are particularly vulnerable. ${ }^{73,75}$ As BCS age, the risk and amount of cognitive decline increases. Any decline in SOP, even if mild, disrupts other cognitive domains (eg, memory) ${ }^{76}$ and interferes with quality of life, autonomy, and everyday functioning. ${ }^{16-18}$ SOP declines are associated with poorer driving performance outcomes, and more at-fault crashes in normal community-dwelling older adults, which represents a growing public health concern. ${ }^{29,77-79}$

\section{SOP Training in BCS (SOAR)}

Fortunately, impairments in SOP can be improved. In a study conducted by Von Ah et al, 10 hours of SOP training over a 2-month period was effective in improving SOP and memory. ${ }^{80}$ In that study, participants completed the SOP training in small groups in a university setting. In the SOP in Middle-Aged and Older Breast Cancer Survivor (SOAR) study, Meneses et al investigated whether these cognitive benefits could be achieved by completing the computerized SOP training individually in a home setting. ${ }^{15}$ In this study, 60 middle-aged and older BCS $\left(M_{\text {age }}=55\right.$ years) who self-reported cognitive impairment were randomized to either the SOP training group $(n=30)$ or a no-contact control group $(n=30)$. Those in the intervention group received up to 10 hours of computerized exercises specifically designed to improve the accuracy and rate in which one can visually process information. Primary outcomes were SOP (Useful Field of View Test), executive function (NIH Toolbox Cognition Battery), and everyday function (Timed Instrumental Activities of Daily Living). Neuropsychological assessments were completed at baseline, 6 weeks, and 6 months post-study entry. These researchers found that those in the SOP training group experienced improvements in SOP, executive function, and everyday function. In this sample, SOP training resulted in improved performance on neuropsychological measures of SOP and executive functioning. Immediate post-test (6 weeks) and 6-month follow-up demonstrated large SOP training effects over time.

\section{Proposal and Conclusion}

Studies on SOP training demonstrate consistent cognitive and everyday functional gains in the short term and long term. ${ }^{16,23,31,38,81}$ The efficacy of these training effects has been observed in various clinical populations, 
including BCS. ${ }^{15}$ Recent work has suggested that SOP training may even reduce the risk of dementia. ${ }^{27}$ As BCS age, they will be in need of cognitive interventions to prevent cognitive loss or remediate for such loss, and SOP training in particular may be adaptive in this clinical population. Figure 1 illustrates that SOP training produces many benefits that address many of the problems BCS experience, especially if they are having cognitive difficulties. BCS often report problems with depression, ${ }^{82,83}$ internal locus of control/motivation, ${ }^{84-86}$ and poor self-rated health and health-related quality of life. ${ }^{87,88}$ Although not documented specifically in the BCS literature, other studies suggest that patients who receive cancer therapy may reduce their driving, especially if they perceive impaired cognitive function. ${ }^{89,90}$

Moving forward, in proposing SOP training with BCS, the following questions should be considered. First, what is the optimal dose needed to provide the most therapeutic benefit to BCS? 10 hours? 20 hours? Second, related to dosage, should booster training be considered to maintain treatment gains? If so, at what time points should booster training be considered. One year, two years, or five years after initial SOP training? Third, what treatment modality or venue would be most efficacious and convenient for BCS? Some may prefer conducting SOP training on their home computer, but for others training in a group setting in a controlled environment may prove to be more therapeutic. Fourth, would pairing SOP training with other techniques known to improve brain health (ie, physical exercise, transcranial direct current stimulation) boost the therapeutic effects? And fifth, what type of BCS participant would benefit the most from SOP training. Cancer treatment, radiation or chemotherapy, may exert different effects on brain health and could impact how effective SOP training may be. Likewise, background characteristics of age, education, income, personality traits (ie, openness, neuroticism) and computer experience could also impact adherence to SOP training. Furthermore, cancer treatments themselves may even reduce depression and anxiety as observed in a study with aromatase inhibitor treatment; ${ }^{83}$ coordination of cancer treatments with SOP training may also produce a variety of treatment outcomes.

Despite the potential for SOP training to address several issues already identified in BCS, no large scale study has been conducted in BCS or any other cancer survivors. This approach represents an innovative and novel approach in which it may not only improve cognitive functioning, but simultaneously improve everyday functioning and quality of life.

\section{Disclosure}

The authors report no conflicts of interest in this work.

\section{References}

1. American Cancer Society. Cancer Facts and Figures 2017. Atlanta, GA: American Cancer Society; 2017.

2. Howlader N, Noone AM, Krapcho M et al. SEER cancer statistics review, 1975-2013 National Cancer Institute. Bethesda, MD: National Cancer Institute; 2016:109-113.

3. Wefel JS, Schagen SB. Chemotherapy-related cognitive dysfunction. Curr Neurol Neurosci Rep. 2012;12(3):267-275. doi:10.1007/ s11910-012-0264-9

4. Janelsins MC, Heckler CE, Peppone LJ, et al. Cognitive complaints in survivors of breast cancer after chemotherapy compared with age-matched controls: an analysis from a nationwide, multicenter, prospective longitudinal study. J Clin Oncol. 2017;35(5):506-514. doi:10.1200/JCO.2016.68.5826

5. Jansen CE, Cooper BA, Dodd MJ, Miaskowski CA. A prospective longitudinal study of chemotherapy-induced cognitive changes in breast cancer patients. Support Care Cancer. 2011;19 (10):1647-1656. doi:10.1007/s00520-010-0997-4

6. Frank JS, Vance DE, Triebel KL, Meneses KM. Cognitive deficits in breast cancer survivors after chemotherapy and hormonal therapy. J Neurosci Nurs. 2015;47(6):302-312. doi:10.1097/ JNN.0000000000000171

7. Koppelmans V, Breteler MM, Boogerd W, Seynaeve C, Gundy C, Schagen SB. Neuropsychological performance in survivors of breast cancer more than 20 years after adjuvant chemotherapy. J Clin Oncol. 2012;30(10):1080-1086. doi:10.1200/JCO.2011.37.0189

8. de Ruiter MB, Reneman L, Boogerd W, et al. Cerebral hyporesponsiveness and cognitive impairment 10 years after chemotherapy for breast cancer. Hum Brain Mapp. 2011;32(8):1206-1219. doi: $10.1002 / \mathrm{hbm} .21102$

9. Wefel JS, Kesler SR, Noll KR, Schagen SB. Clinical characteristics, pathophysiology, and management of noncentral nervous system cancer-related cognitive impairment in adults. CA Cancer J Clin. 2015;65(2):123-138. doi:10.3322/caac. 21258

10. Bower JE. Behavioral symptoms in patients with breast cancer and survivors. J Clin Oncol. 2008;26(5):768-777. doi:10.1200/ JCO.2007.14.3248

11. Hodgson KD, Hutchinson AD, Wilson CJ, Nettelbeck T. A meta-analysis of the effects of chemotherapy on cognition in patients with cancer. Cancer Treat Rev. 2013;39(3):297-304. doi:10.1016/j. ctrv.2012.11.001

12. Ahles TA, Saykin A. Cognitive effects of standard-dose chemotherapy in patients with cancer. Cancer Invest. 2001;19(8):812-820. doi:10.1081/CNV-100107743

13. Ahles TA, Saykin AJ, Furstenberg CT, et al. Neuropsychologic impact of standard-dose systemic chemotherapy in long-term survivors of breast cancer and lymphoma. J Clin Oncol. 2002;20 (2):485-493. doi:10.1200/JCO.2002.20.2.485

14. Wefel JS, Kayl AE, Meyers CA. Neuropsychological dysfunction associated with cancer and cancer therapies: a conceptual review of an emerging target. $\mathrm{Br} \quad J$ Cancer. 2004;90(9):1691-1696. doi:10.1038/sj.bjc. 6601772

15. Meneses K, Benz R, Bail JR, et al. Speed of processing training in middle-aged and older breast cancer survivors (SOAR): results of a randomized controlled pilot. Breast Cancer Res Treat. 2018;168 (1):259-267. doi:10.1007/s10549-017-4564-2 
16. Ball K, Berch DB, Helmers KF, et al. Effects of cognitive training interventions with older adults: a randomized controlled trial. JAMA. 2002;288(18):2271-2281. doi:10.1001/jama.288.18.2271

17. Ball K, Edwards JD, Ross LA, McGwin G. Cognitive training decreases motor vehicle collision involvement of older drivers. $J$ Am Geriatr Soc. 2010;58(11):2107-2113. doi:10.1111/j.15325415.2010.03138. $\mathrm{x}$

18. Ball K, Edwards JD, Ross LA. The impact of speed of processing training on cognitive and everyday functions. J Gerontol B Psychol Sci Soc Sci. 2007;62:19-31. doi:10.1093/geronb/62. special_issue_1.19

19. Vance DE, Crowe M. A proposed model of neuroplasticity and cognitive reserve in older adults. Act Adapt Aging. 2006;30 (3):61-79. doi:10.1300/J016v30n03_04

20. Vance DE, Wright MA. Positive and negative neuroplasticity: implications for age-related cognitive declines. J Gerontol Nurs. 2009;35 (6):11-17. doi:10.3928/00989134-20090428-02

21. Vance DE, Roberson AJ, McGuinness TM, Fazeli PL. How neuroplasticity and cognitive reserve protect cognitive functioning. $J$ Psychosoc Nurs Ment Health Serv. 2010;48(4):23-30. doi:10.3928/02793695-20100302-01

22. Vance DE, Kaur J, Fazeli PL, et al. Neuroplasticity and successful cognitive aging: a brief overview for nursing. J Neurosci Nurs. 2012;44(4):218-227. doi:10.1097/JNN.0b013e3182527571

23. Wadley VG, Benz RL, Ball KK, Roenker DL, Edwards JD, Vance DE. Development and evaluation of home-based speed-ofprocessing training for older adults. Arch Phys Med Rehabil. 2006;87(6):757-763. doi:10.1016/j.apmr.2006.02.027

24. Vance DE, McNees P, Meneses K. Technology, cognitive remediation, and nursing: directions for successful cognitive aging. $J$ Gerontol Nurs. 2009;35(2):50-56.

25. Lampit A, Hallock H, Valenzuela M. Computerized cognitive training in cognitively healthy older adults: a systematic review and meta-analysis of effect modifiers. PLoS Med. 2014;11(11): e1001756. doi:10.1371/journal.pmed.1001756

26. Rebok GW, Ball K, Guey LT, et al. Ten-year effects of the advanced cognitive training for independent and vital elderly cognitive training trial on cognition and everyday functioning in older adults. $J \mathrm{Am}$ Geriatr Soc. 2014;62(1):16-24. doi:10.1111/jgs.12607

27. Edwards JD, Xu H, Clark DO, Guey LT, Ross LA, Unverzagt FW. Speed of processing training results in lower risk of dementia. Alzheimers Dement. 2017;3(4):603-611. doi:10.1016/j. trci.2017.09.002

28. O'Connor ML, Hudak EM, Edwards JD. Cognitive speed of processing training can promote community mobility among older adults: a brief review. J Aging Res. 2011;2011:430802. doi:10.4061/2011/ 430802

29. Edwards JD, Myers C, Ross LA, et al. The longitudinal impact of cognitive speed of processing training on driving mobility. Gerontologist. 2009;49(4):485-494. doi:10.1093/geront/gnp042

30. Ross LA, Edwards JD, O'Connor ML, Ball KK, Wadley VG, Vance DE. The transfer of cognitive speed of processing training to older adults' driving mobility across 5 years. J Gerontol B Psychol Sci Soc Sci. 2016;71(1):87-97. doi:10.1093/geronb/gbv022

31. Vance DE, Dawson J, Wadley VG, et al. The accelerate study: the longitudinal effect of speed of processing training on cognitive performance of older adults. Rehabil Psychol. 2007;51(1):89-96. doi:10.1037/0090-5550.52.1.89

32. Edwards JD, Valdes EG, Peronto C, et al. The efficacy of insight cognitive training to improve useful field of view performance: a brief report. J Gerontol B Psychol Sci Soc Sci. 2015;70 (3):417-422. doi:10.1093/geronb/gbt113

33. Cabeza R, Nyberg L. Imaging cognition II: an empirical review of 275 PET and fMRI studies. $J$ Cogn Neurosci. 2000;12(1):1-47. doi: $10.1162 / 08989290051137585$
34. O’Brien JL, Edwards JD, Maxfield ND, Peronto CL, Williams VA, Lister JJ. Cognitive training and selective attention in the aging brain: an electrophysiological study. Clin Neurophysiol. 2013;124 (11):2198-2208. doi:10.1016/j.clinph.2013.05.012

35. Kesler SR, Watson C, Koovakkattu D, et al. Elevated prefrontal myo-inositol and choline following breast cancer chemotherapy. Brain Imaging Behav. 2013;7(4):501-510. doi:10.1007/s11682-0139228-1

36. Kesler SR, Kent JS, O'Hara R. Prefrontal cortex and executive function impairments in primary breast cancer. Arch Neurol. 2011;68(11):1447-1453. doi:10.1001/archneurol.2011.245

37. Ross LA, Webb CE, Whitaker C, et al. The effects of useful field of view training on brain activity and connectivity. J Gerontol B Psychol Sci Soc Sci. 2018

38. Edwards JD, Wadley VG, Vance DE, Wood K, Roenker DL, Ball KK. The impact of speed of processing training on cognitive and everyday performance. Aging Ment Health. 2005;9(3):262-271. doi: $10.1080 / 13607860412331336788$

39. Wolinsky FD, Vander Weg MW, Howren MB, Jones MP, Dotson MM. The effect of cognitive speed of processing training on the development of additional IADL difficulties and the reduction of depressive symptoms: results from the IHAMS randomized controlled trial. J Aging Health. 2015;27(2):334-354. doi:10.1177/ 0898264314550715

40. Ross LA, Freed SA, Edwards JD, Phillips CB, Ball K. The impact of three cognitive training programs on driving cessation across 10 years: a randomized controlled trial. Gerontologist. 2017;57 (5):838-846. doi:10.1093/geront/gnw143

41. Wolinsky FD, Vander Weg MW, Martin R, et al. The effect of speed-of-processing training on depressive symptoms in ACTIVE. J Gerontol a Biol Sci Med Sci. 2009;64A(4):468-472. doi:10.1093/ gerona/gln044

42. Wolinsky FD, Vander Weg MW, Martin R, et al. Does cognitive training improve internal locus of control among older adults? $J$ Gerontol B Psychol Sci Soc Sci. 2010;65(5):591-598. doi:10.1093/geronb/gbp117

43. Astin JA, Shapiro J, Shapiro D. Psychological control and morbidity/ mortality in breast cancer patients: a 20-year follow-up study. Behav Med. 2013;39(1):7-10. doi:10.1080/08964289.2012.708683

44. Wolinsky FD, Mahncke H, Vander Weg MW, et al. Speed of processing training protects self-rated health in older adults: enduring effects observed in the multi-site ACTIVE randomized controlled trial. Int Psychogeriatr. 2010;22(3):470-478. doi:10.1017/ S1041610209991281

45. Wolinsky FD, Unverzagt FW, Smith DM, Jones R, Wright E, Tennstedt SL. The effects of the ACTIVE cognitive training trial on clinically relevant declines in health-related quality of life. J Gerontol B Psychol Sci Soc Sci. 2006;61(5):S281-287. doi:10.1093/geronb/ 61.5.S281

46. Wolinsky FD, Unverzagt FW, Smith DM, Jones R, Stoddard A, Tennstedt SL. The ACTIVE cognitive training trial and health-related quality of life: protection that lasts for 5 years. $J$ Gerontol a Biol Sci Med Sci. 2006;61(12):1324-1329. doi:10.1093/gerona/61.12.1324

47. Gold PE. Acetylcholine modulation of neural systems involved in learning and memory. Neurobiol Learn Mem. 2003;80(3):194-210. doi:10.1016/j.nlm.2003.07.003

48. Wise RA. Dopamine, learning and motivation. Nat Rev Neurosci. 2004;5(6):483-494. doi:10.1038/nrn1406

49. Wise RA. Rewards wanted: molecular mechanisms of motivation. Discov Med. 2004;4(22):180-186.

50. Edwards J, Wadley V, Myers R, Roenker DL, Cissell G, Ball K. Transfer of a speed of processing intervention to near and far cognitive functions. Gerontology. 2002;48(5):329-340. doi:10.1159/ 000065259 
51. Vance DE, Fazeli PL, Ross LA, Wadley VG, Ball KK. Speed of processing training with middle-age and older adults with HIV: a pilot study. J Assoc Nurses AIDS Care. 2012;23(6):500-510. doi:10.1016/j.jana.2012.01.005

52. Vance D, Fazeli P, Shacka J, et al. Testing a computerized cognitive training protocol in adults aging with HIV-associated neurocognitive disorders: randomized controlled trial rationale and protocol. JMIR Res Protoc. 2017;6(4):e68. doi:10.2196/resprot.6625

53. Seigers R, Fardell JE. Neurobiological basis of chemotherapy-induced cognitive impairment: a review of rodent research. Neurosci Biobehav Rev. 2011;35(3):729-741. doi:10.1016/ j.neubiorev.2010.09.006

54. Janelsins MC, Kesler SR, Ahles TA, Morrow GR. Prevalence, mechanisms, and management of cancer-related cognitive impairment. Int Rev Psychiatry. 2014;26(1):102-113. doi:10.3109/ 09540261.2013.864260

55. Baudino B, D'Agata F, Caroppo P, et al. The chemotherapy long-term effect on cognitive functions and brain metabolism in lymphoma patients. Q J Nucl Med Mol Imaging. 2012;56(6):559-568.

56. Adams-Price CE, Morse LW, Cross GW, Williams M, Wells-Parker E. The effects of chemotherapy on useful field of view (UFOV) in younger and older breast cancer patients. Exp Aging Res. 2009;35 (2):220-234. doi:10.1080/03610730902720497

57. Collins B, MacKenzie J, Tasca GA, Scherling C, Smith A. Cognitive effects of chemotherapy in breast cancer patients: a dose-response study. Psychooncology. 2013;22(7):1517-1527. doi:10.1002/ pon. 3163

58. Hurria A, Rosen C, Hudis C, et al. Cognitive function of older patients receiving adjuvant chemotherapy for breast cancer: a pilot prospective longitudinal study. $J$ Am Geriatr Soc. 2006;54 (6):925-931. doi:10.1111/j.1532-5415.2006.00732.x

59. Ahles TA, Saykin AJ, McDonald BC, et al. Longitudinal assessment of cognitive changes associated with adjuvant treatment for breast cancer: impact of age and cognitive reserve. J Clin Oncol. 2010;28 (29):4434-4440. doi:10.1200/JCO.2009.27.0827

60. Tavan H, Azadi A, Veisani Y. Return to work in cancer patients: a systematic review and meta-analysis. Indian $J$ Palliat Care. 2019;25(1):147-152. doi:10.4103/IJPC.IJPC_114_18

61. Von Ah D, Storey S, Crouch A. Relationship between self-reported cognitive function and work-related outcomes in breast cancer survivors. J Cancer Surviv. 2018;12(2):246-255. doi:10.1007/ s11764-017-0664-6

62. Sun Y, Shigaki CL, Armer JM. Return to work among breast cancer survivors: a literature review. Support Care Cancer. 2017;25 (3):709-718. doi:10.1007/s00520-016-3446-1

63. Mathias JL, Lucas LK. Cognitive predictors of unsafe driving in older drivers: a meta-analysis. Int Psychogeriatr. 2009;21 (4):637-653. doi:10.1017/S1041610209009119

64. Loh KP, Janelsins MC, Mohile SG, et al. Chemotherapy-related cognitive impairment in older patients with cancer. J Geriatr Oncol. 2016;7(4):270-280. doi:10.1016/j.jgo.2016.04.008

65. Lindner O, Phillips B, McCabe M, et al. A meta-analysis of cognitive impairment following adult cancer chemotherapy. Neuropsychology. 2014;28(5):726-740. doi:10.1037/neu0000064

66. Ahles TA, Root JC, Ryan EL. Cancer- and cancer treatment-associated cognitive change: an update on the state of the science. J Clin Oncol. 2012;30(30):3675-3686. doi:10.1200/ JCO.2012.43.0116

67. Sanoff HK, Deal AM, Krishnamurthy J, et al. Effect of cytotoxic chemotherapy on markers of molecular age in patients with breast cancer. J Natl Cancer Inst. 2014;106(4):dju057. doi:10.1093/jnci/ dju057

68. Menning S, de Ruiter MB, Veltman DJ, et al. Changes in brain white matter integrity after systemic treatment for breast cancer: a prospective longitudinal study. Brain Imaging Behav. 2018;12 (2):324-334. doi:10.1007/s11682-017-9695-x
69. Koppelmans V, de Groot M, de Ruiter MB, et al. Global and focal white matter integrity in breast cancer survivors 20 years after adjuvant chemotherapy. Hum Brain Mapp. 2014;35(3):889-899. doi:10.1002/hbm.22221

70. Kesler SR, Rao V, Ray WJ, Rao A. Alzheimer's disease neuroimaging I. Probability of Alzheimer's disease in breast cancer survivors based on gray-matter structural network efficiency. Alzheimers Dement. 2017;9:67-75. doi:10.1016/j.dadm.2017.10.002

71. Schuitema I, Deprez S, Van Hecke W, et al. Accelerated aging, decreased white matter integrity, and associated neuropsychological dysfunction 25 years after pediatric lymphoid malignancies. $J$ Clin Oncol. 2013;31(27):3378-3388. doi:10.1200/JCO.2012.46.7050

72. Fried LP, Tangen CM, Walston J, et al. Frailty in older adults: evidence for a phenotype. J Gerontol a Biol Sci Med Sci. 2001;56 (3):M146-156. doi:10.1093/gerona/56.3.M146

73. Mandelblatt JS, Small BJ, Luta G, et al. Cancer-related cognitive outcomes among older breast cancer survivors in the thinking and living with cancer study. J Clin Oncol. 2018;36:3211.

74. Mandelblatt JS, Stern RA, Luta G, et al. Cognitive impairment in older patients with breast cancer before systemic therapy: is there an interaction between cancer and comorbidity? J Clin Oncol. 2014;32 (18):1909-1918. doi:10.1200/JCO.2013.54.2050

75. Mandelblatt JS, Jacobsen PB, Ahles T. Cognitive effects of cancer systemic therapy: implications for the care of older patients and survivors. J Clin Oncol. 2014;32(24):2617-2626. doi:10.1200/ JCO.2014.55.1259

76. Clay OJ, Edwards JD, Ross LA, et al. Visual function and cognitive speed of processing mediate age-related decline in memory span and fluid intelligence. J Aging Health. 2009;21(4):547-566. doi:10.1177/ 0898264309333326

77. Wadley VG, Okonkwo O, Crowe M, et al. Mild cognitive impairment and everyday function: an investigation of driving performance. $J$ Geriatr Psychiatry Neurol. 2009;22(2):87-94. doi:10.1177/ 0891988708328215

78. Vance DE, Roenker DL, Cissell GM, Edwards JD, Wadley VG, Ball KK. Predictors of driving exposure and avoidance in a field study of older drivers from the state of Maryland. Accid Anal Prev. 2006;38(4):823-831. doi:10.1016/j.aap.2006.02.008

79. Friedman C, McGwin G, Ball KK, Owsley C. Association between higher-order visual processing abilities and a history of motor vehicle collision involvement by drivers ages 70 and over. Invest Ophthalmol Vis Sci. 2013;54(1):778. doi:10.1167/iovs.12-11249

80. Von Ah D, Carpenter JS, Saykin A, et al. Advanced cognitive training for breast cancer survivors: a randomized controlled trial. Breast Cancer Res Treat. 2012;135(3):799-809. doi:10.1007/s10549-0122210-6

81. Ball KK, Ross LA, Roth DL, Edwards JD. Speed of processing training in the ACTIVE study: how much is needed and who benefits? J Aging Health. 2013;25(8Suppl):65S-84S. doi:10.1177/ 0898264312470167

82. Puigpinos-Riera R, Graells-Sans A, Serral G, et al. Anxiety and depression in women with breast cancer: social and clinical determinants and influence of the social network and social support (DAMA cohort). Cancer Epidemiol. 2018;55:123-129. doi:10.1016/j. canep.2018.06.002

83. Martino G, Catalano A, Agostino RM, et al. Quality of life and psychological functioning in postmenopausal women undergoing aromatase inhibitor treatment for early breast cancer. PLoS One. 2020;15(3):e0230681. doi:10.1371/journal.pone.0230681

84. Milne HM, Wallman KE, Guilfoyle A, Gordon S, Corneya KS. Selfdetermination theory and physical activity among breast cancer survivors. J Sport Exerc Psychol. 2008;30(1):23-38. doi:10.1123/ jsep.30.1.23

85. Yi M, Kim J. Factors influencing health-promoting behaviors in Korean breast cancer survivors. Eur J Oncol Nurs. 2013;17 (2):138-145. doi:10.1016/j.ejon.2012.05.001 
86. Garcia-Jimenez M, Santoyo-Olsson J, Ortiz C, Lahiff M, SokalGutierrez K, Napoles AM. Acculturation, inner peace, cancer self-efficacy, and self-rated health among Latina breast cancer survivors. J Health Care Poor Underserved. 2014;25(4):1586-1602. doi:10.1353/hpu.2014.0158

87. Daldoul A, Khechine W, Bhiri H, et al. Factors predictive of quality of life among breast cancer patients. Asian Pac J Cancer Prev. 2018;19(6):1671-1675. doi:10.22034/APJCP.2018.19.6.1671

88. Schmidt ME, Wiskemann J, Steindorf K. Quality of life, problems, and needs of disease-free breast cancer survivors 5 years after diagnosis. Qual Life Res. 2018;27(8):2077-2086. doi:10.1007/ s11136-018-1866-8
89. Yuen HK, Gillespie MB, Day TA, Morgan L, Burik JK. Driving behaviors in patients with head and neck cancer during and after cancer treatment: a preliminary report. Head Neck. 2007;29 (7):675-681. doi:10.1002/hed.20567

90. Yuen HK, Gillespie MB, Barkley RA, Day TA, Bandyopadhyay D, Sharma AK. Driving performance in patients with cancer in the head and neck region: a pilot study. Arch Otolaryngol Head Neck Surg. 2007;133(9):904-909. doi:10.1001/archotol.133.9.904

\section{Publish your work in this journal}

Nursing: Research and Reviews is an international, peer-reviewed, open access journal publishing original research, reports, reviews and commentaries on all aspects of nursing and patient care. These include patient education and counseling, ethics, management and organizational issues, diagnostics and prescribing, health outcomes, economics and resource management, improving patient safety in all settings. The manuscript management system is completely online and includes a very quick and fair peer-review system. Visit http://www. dovepress.com/testimonials.php to read real quotes from published authors. 